EPJ Web of Conferences 73, 02006 (2014)

DOI: $10.1051 /$ epjconf/20147302006

(C) Owned by the authors, published by EDP Sciences, 2014

\title{
Measurement of TMD observables at COMPASS
}

\author{
Giulio Sbrizzai ${ }^{1,2, a}$ on behalf of the COMPASS Collaboration \\ ${ }^{1}$ Trieste University, Physics Department, Italy \\ ${ }^{2}$ INFN Trieste, Italy
}

\begin{abstract}
COMPASS is a fixed target experiment at the CERN SPS taking data since 2002. An important part of the physics programme is the study of the spin structure of the nucleon by measuring deep inelastic scattering of high energy muons off polarized or unpolarized nucleons. In particular COMPASS has given and is giving an important contribution to understand the role of parton transverse spin and transverse momentum, a recent topic on which a large international community is working. The most recent COMPASS measurements in this field and their relevance are here reviewed.
\end{abstract}

\section{Introduction}

The description of the partonic structure of the nucleon is one of the central problems of hadronic physics. In recent years considerable theoretical and experimental progress has been made and the relevance of the quark transverse spin and transverse momentum has been clearly assessed. In the present theoretical framework, eight transverse momentum dependent parton distribution functions (TMD PDFs) are required at leading order in the one photon-exchange approximation. They describe all possible correlations between the transverse momentum and spin of the quarks and the spin of the nucleon.

Experimental data from the semi-inclusive deeply inelastic lepton scattering (SIDIS) are today the major source of information to access the TMD PDFs. They allow to access easily convolutions of the differerent TMD PDFs and fragmentation functions via high statistic measurements of asymmetries in the azimuthal distributions of the final state hadrons.

COMPASS (COmmon Muon and Proton Apparatus for Structure and Spectroscopy) is a fixed target experiment at the CERN SPS taking data since 2002. A detailed description of the COMPASS apparatus is found in [1]. An important part of the experimental programme consists in the study of the nucleon structure and SIDIS data have been collected using a $160 \mathrm{GeV}$ longitudinally polarized muon beam and either longitudinally or transversely polarized proton $\left(\mathrm{NH}_{3}\right)$ and deuteron $\left({ }^{6} \mathrm{LiD}\right)$ targets. All the terms appearing in the polarised SIDIS cross section are measured in COMPASS. A selection of the results is presented here, with particular focus on the results which allow to assess the relevance of the role played by the TMD PDFs in the nucleon description.

\footnotetext{
ae-mail: giulio.sbrizzai@ts.infn.it
}

This is an Open Access article distributed under the terms of the Creative Commons Attribution License 4.0, which permits unrestricted use, distribution, and reproduction in any medium, provided the original work is properly cited. 


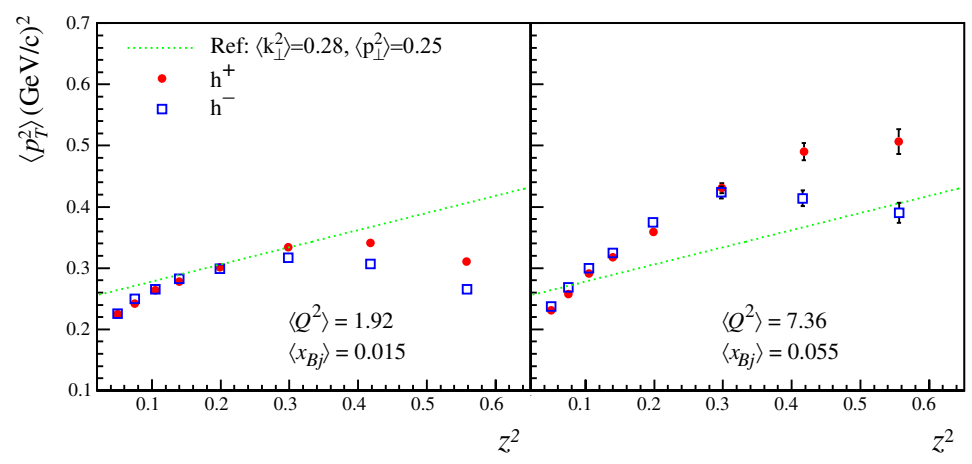

Figure 1. $\left\langle\left(p_{T}^{h}\right)^{2}\right\rangle$ vs $z^{2}$ for two $\left(Q^{2}, x\right)$ intervals. The corresponding mean values $\left\langle Q^{2}\right\rangle$ (in units of $(G e V / c)^{2}$ ) and $\langle x\rangle$ are indicated in the figure. The dotted green line corresponds to the realtion $\left\langle\left(p_{T}^{h}\right)^{2}\right\rangle=\left\langle p_{\perp}^{2}\right\rangle+z^{2}\left\langle k_{\perp}^{2}\right\rangle$ with constant $\left\langle p_{\perp}^{2}\right\rangle$ and $\left\langle k_{\perp}^{2}\right\rangle$ from reference [2].

I start this contribution by showing in Sect. 2.1 the results extracted from the measurements of the transverse momentum dependence of the unpolarised hadron multiplicities.

In Sect. 2.2, the unpolarised azimuthal asymmetries of charged hadrons are presented. They are driven by the Cahn effect and the Boer-Mulders (B-M) TMD PDF.

Among all the expected transverse spin dependent azimuthal asymmetries I will address in Sect. 3 only the one related to the Sivers function.

\section{Results}

\subsection{Transverse momentum distribution in un-polarised SIDIS}

The transverse momentum of the hadron with respect to the virtual photon direction $p_{T}^{h}$ is given by the sum of the instrinsic transverse momentum of the quark $k_{\perp}$ and of the transverse momentum $p_{\perp}$ the hadron gets in the fragmentation process. Using a simple model [2] one expect $\left\langle\left(p_{T}^{h}\right)^{2}\right\rangle=\left\langle p_{\perp}^{2}\right\rangle+z^{2}\left\langle k_{\perp}^{2}\right\rangle$ where $\left\langle\left(p_{T}^{h}\right)^{2}\right\rangle$ is the $\left(p_{T}^{h}\right)^{2}$ mean value calculated in a given kinematical bin and $z$ is the hadron's fraction of the available energy. The $\left\langle\left(p_{T}^{h}\right)^{2}\right\rangle$ values are calculated from the measured $\left(p_{T}^{h}\right)^{2}$ charged hadrons distributions corrected by the apparatus acceptance estimated using Monte Carlo simulation. Their dependency versus $z^{2}$ is studied in a two-dimensional grid binning both on the Bjorken scaling variable $x$ and on the photon virtuality $Q^{2}$. Results are shown in Fig. 1 for positive and negative hadrons separately.

These precise measurements [3] are very interesting since they show a deviation from the expected linear behaviour, shown by the dotted green line in Fig. 1. They also allow to study in detail possible kinematical dependencies of the transverse momentum of the quark.

\subsection{Azimuthal asymmetries in unpolarised SIDIS}

The study of the $\cos \left(\phi_{h}\right)$ and $\cos \left(2 \phi_{h}\right)$ modulations, where $\phi_{h}$ is the azimuthal angle of the hadron around the virtual photon direction, is of particular interest in the framework of the TMD approach to the PDFs. The amplitudes of these modulations, $A_{\cos \phi_{h}}^{U U}$ and $A_{\cos 2 \phi_{h}}^{U U}$, are both expected to come from the kinematic of the scattering process (Cahn effect) and from the correlation between $k_{T}$ and the transverse spin. Such a correlation is encoded in the Boer-Mulders TMD PDF, which appears convoluted with the famous Collins Fragmentation Function. An $A_{\sin \phi_{h}}^{L U}$ asymmetry is also expected and has been measured, 


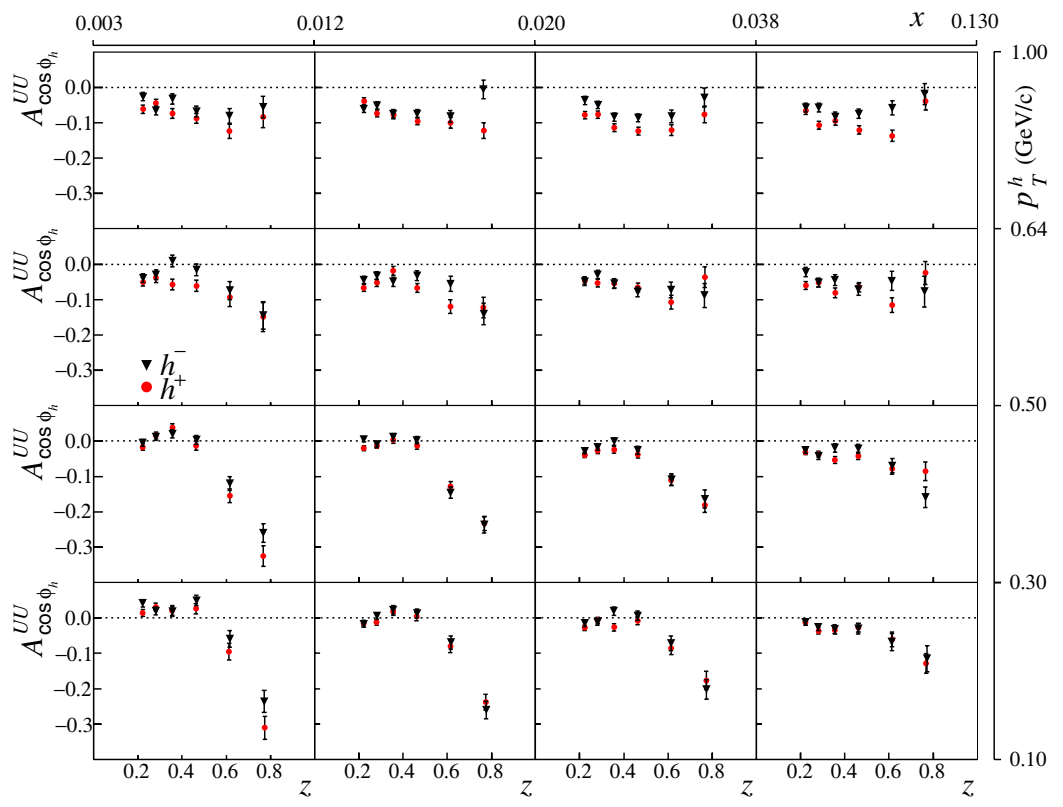

Figure 2. COMPASS results on the spin-averaged azimuthal asymmetries from positive (red) and negative (black) hadrons. The amplitudes $A_{\text {cos } \phi_{h}}^{U U}$ of the $\cos \left(\phi_{h}\right)$ asymmetries are shown as function of $z$ in the different $x$ and $p_{T}^{h}$ bins. The systematic uncertainties are estimated to be twice the statistical errors.

but it turns out to be small. It is given by higher twist effects and has no clear interpretation in terms of the parton model.

The measured azimuthal distributions are corrected by the $\phi_{h}$ dependent part of the apparatus acceptance and fitted with the appropriate $\phi_{h}$ function. Many studies have been done to optimise the Monte Carlo description of the experimental apparatus and different parametrisations have been used to estimate the systematic uncertainties.

These asymmetries are extracted from data collected in 2004 on a deuteron target. The asymmetries are given both separately as a function of the relevant kinematic variables $\left(x, z\right.$ or $\left.p_{T}^{h}\right)$ and in a threedimensional grid of these three variables. The amplitudes $A_{\cos \phi_{h}}^{U U}$ and $A_{\cos 2 \phi_{h}}^{U U}$ show strong kinematic dependences both for positive and negative hadrons, as shown in Fig. 2 where $A_{\cos \phi_{h}}^{U U}$ is extracted in the three-dimensional grid. Also $A_{\cos 2 \phi_{h}}^{U U}$ shows a similar strong dependence on the $x, z$ and $p_{T}^{h}$ variables, which up to now has not been reproduced by theoretical models.

\section{Sivers TMD PDF}

The Sivers function correlates $k_{\perp}$ with the transverse polarisation of the nucleon and is related to the orbital angular momentum of the quarks inside the nucleon. It gives rise to a modulation of the type $\sin \left(\phi_{h}-\phi_{S}\right)$ in the polarised SIDIS cross section, where $\phi_{S}$ is the azimuthal angle of the transverse target polarisation around the virtual photon direction.

The precise measurements of the amplitude $A_{\text {Siv }}$ obtained from the data collected on transversely polarised protons in two years of data taking (2007 [4] and 2010 [5] is shown in Fig. 3). The data exhibit a clear positive signal for positive hadrons, which is small in the low $x$ region and rather large in the quark valence region, where the corresponding $Q^{2}$ values are larger. The high statistic of the data 


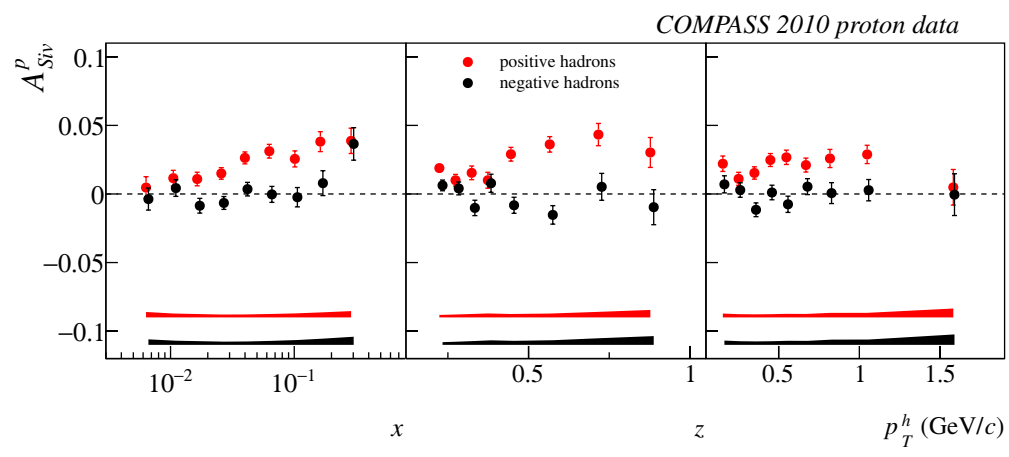

Figure 3. Sivers asymmetries for positive (red) and negative (black) hadrons as functions of $x, z$ and $p_{T}^{h}$ from the 2010 proton data.

collected and the large measured interval in $Q^{2}$ give also the possibility to perform a multi dimensional analysis and to study the $Q^{2}$ TMD evolution.

\section{Conlusion and outlook}

The SIDIS polarised azimuthal cross section is a very useful tool to access the TMD PDFs, and the particular $x$ and $Q^{2}$ ranges explored by COMPASS is complementary to other experiments. Interesting and challenging kinematic dependencies have been found in the unpolarised azimuthal asymmetries and in the $p_{T}^{h}$ depencence of the hadron multiplicities. The asymmetries related to the Sivers function and the Collins functions are clearly visible while the other TMD related six asymmetries expected to appear in the target transverse polarisation dependent part of the SIDIS cross section are measured to compatible with zero.

More results are coming from the multi dimensional analysis for the azimuthal asymmetries in SIDIS off transversely polarised target from the 2010 proton data. Also, precise measurements from SIDIS on a long liquid $\mathrm{H} 2$ target will be performed in parallel to the DVCS measurement and complementary information on the Sivers and B-M TMD PDFs will come from polarised Drell-Yan from COMPASS II (2014-2017).

\section{References}

[1] P. Abbon et al. [COMPASS Collaboration], Nucl. Instrum. Meth. A 577, 455 (2007)

[2] M. Anselmino, M. Boglione, A. Prokudin, C. Turk, Eur. Phys. J. A 31, 373 (2007)

[3] C. Adolph et al. [COMPASS Collaboration], Eur. Phys. J. C 73, 2531 (2013)

[4] M.G. Alekseev et al. [COMPASS Collaboration], Phys. Lett. B 692, 240 (2010)

[5] C. Adolph et al. [COMPASS Collaboration], Phys. Lett. B 717, 383 (2012)

[6] A.V. Efremov, P. Schweitzer, O.V. Teryaev and P. Zavada, Phys. Rev. D 80, 014021 (2009) 